\title{
Philosophiques
}

\section{Behaviorisme et psychologie}

\section{Michael Schleifer}

Volume 4, numéro 2, octobre 1977

Philosophie et psychologie

URI : https://id.erudit.org/iderudit/203083ar

DOI : https://doi.org/10.7202/203083ar

Aller au sommaire du numéro

Éditeur(s)

Société de philosophie du Québec

ISSN

0316-2923 (imprimé)

1492-1391 (numérique)

Découvrir la revue

Citer cet article

Schleifer, M. (1977). Behaviorisme et psychologie. Philosophiques, 4(2), 327-334.

https://doi.org/10.7202/203083ar d'utilisation que vous pouvez consulter en ligne.

https://apropos.erudit.org/fr/usagers/politique-dutilisation/ 


\title{
BEHAVIORISME ET PSYCHOLOGIE
}

\author{
par Michael Schleifer
}

Je ne veux pas répéter les arguments maintenant familiers contre le behaviorisme méthodologique, résumés d'une façon excellente dans la présentation de M. Kaufmann ${ }^{1}$. Je voudrais néanmoins porter emphase sur un des aspects de son analyse de Chomsky ${ }^{2}$. Ce facteur contredisant les vues de Skinner tient au fait que le langage est illimité, c'est-à-dire que nous avons la " compétence " (de même qu'un enfant de deux ans) de créer de nouvelles phrases jamais entendues ou utilisées auparavant. Cet argument de Chomsky peut être opposé à toute autre forme de behaviorisme.

Je me propose dans une première partie de commenter le behaviorisme mais surtout le "logical behaviorism ", espérant que le tout complétera les commentaires de $\mathbf{M}$. Kaufmann. Dans un second temps, j'amorcerai la discussion sur les recherches en psychologie développementale, plus particulièrement en ce qui concerne la moralité et la causalité. Je souhaiterais démontrer comment une orientation développementale s'oppose à l'approche d'entraînement des behavioristes modernes. A ce moment, je soulignerai les raisons qui me font m'objecter contre cette forme ou toute autre de behaviorisme.

Le mot "behavioriste" est très ambigu et un psychologue voulant en être adepte doit choisir parmi une multitude d'orientations. $\grave{A}$ une extrémité on retrouve l'affirmation du fameux philosophe, W.V. Quine, voulant que « tout homme raisonnable est un behavioriste $"^{3}$. Pour ce dernier, le behaviorisme en psychologie

1. Psychologie de la "conscience" et "science du behavior".

2. Ibid, page 322.

3. Linguistics and Pbilosopby (rédaction S. Hook), 1968, p. 27. 
n'est qu'un exemple du point de vue empiriste selon lequel les conjectures et les conclusions doivent être éventuellement vérifiées. Beaucoup d'antibehavioristes accepteraient cette définition sans difficulté parce qu'elle englobe d'une façon généralisatrice des principes scientifiques incontestables. S'il n'en tient qu'à cela, Piaget et Chomsky adhèrent au mouvement behavioriste puisqu'ils soutiennent non seulement les principes énoncés plus haut, mais aussi qu'on devrait viser à une classification rationnelle des événements observés, qu'on devrait analyser les interdépendances des événements significatifs et utiliser les méthodes statistiques et mathématiques si elles sont applicables et pertinentes.

Nous savons pertinemment qu'ils n'en sont pas puisqu'ils utilisent consciemment des concepts n'ayant aucun lien logique avec des comportements. Selon Fodor ${ }^{4}$, pour être qualifié de behavioriste, on doit être adepte de la seule affirmation suivantes:

"For each mental predicate that can be employed in a psychological explanation, there must be at least one description of beha. viour to which it bears a logical connection."

Nous justifions l'emploi de certains concepts parce que, selon nous, ils sont les seuls à pouvoir expliquer certains phénomènes. $C^{\prime}$ 'est ce qui annule les attaques de MacCorquodale ${ }^{6}$ concernant les structures inconscientes (deep structure) dans la théorie de Chomsky, et celles de Bandura ${ }^{7}$ concernant la théorie des stades de Piaget. Ces théories ne sont soumises ni à un "operationalism " qui veut que chaque concept puisse être mesuré physiquement, ni à l'argument déjà énoncé précédemment qui veut que tout concept soit logiquement lié à une réponse comportementale. De la même façon que Chomsky justifie sa théorie par la structure du langage, Piaget peut revendiquer la nécessité de sa théorie à cause de la structure de la pensée de l'enfant.

Bien que le débat se soit déroulé surtout en psychologie, la majorité des appuis au behaviorisme venait de philosophes, dont

\footnotetext{
4. An Introduction to the Philosopby of Psychology, 1968, Random House Ibid., p. 51.

"Behaviourism is a Humanism ", Without/Witbin (Rédaction Matson), Wadsworth, 1973.

7. "Social Learning of Moral Judgments ", Journal of Personality and Social Psychology., 1969,11 , pp. $275-279$.
} 
Wittgenstein ${ }^{8}$, Ryle , et Malcolm ${ }^{10}$. Ce groupe, appelé « logical behaviorist ", voulait démontrer que les concepts mentaux étaient nécessairement liés à un comportement quelconque, non pour des raisons méthodologiques mais pour des considérations linguistiques. Selon Malcolm ${ }^{11}$ par exemple, le rêve existe mais ne peut être oublié, car la question : "Est-ce que le rêveur se rappelle ou oublie son rêve? " n'a pas de sens. En effet, selon lui, le critère justifiant l'existence d'un rêve est le rapport qu'on en fait. Cette théorie linguistique affirme qu'un enfant doit apprendre ce qu'est un rêve en le racontant. En opposition à cette théorie du langage que l'on doit réfuter, Fodor ${ }^{12}$ a prouvé qu'on organise différemment notre langage. Ainsi, on peut dire de quelqu'un qu'il rêve, conformément à notre théorie des images mentales. Les enfants peuvent facilement apprendre de cette façon ce qu'est un rêve, (une image mentale pendant qu'ils dorment), et nous pouvons ignorer par ce fait les critères comportementaux. Si nous n'avons pas à relier nos événements quotidiens à des critères de comportement, il peut en être de même pour nos explications psychologiques.

En démontrant comme nous venons de le faire que l'appui philosophique est inadéquat, on espère qu'en s'en rendant compte, les psychologues n'adhéreront plus au mouvement behavioriste, surtout en ce qui concerne la psychologie développementale où il est le moins approprié. Le principal défaut de l'épistémologie du behaviorisme pour la psychologie enfantine, c'est qu'il ne permet pas au psychologue de considérer les processus cognitifs comme nécessitant des connaissances. Or, la contribution fondamentale de Piaget en psychologie développementale est d'insister sur le fait qu'on doive observer le développement de l'enfant en terme des "catégories" philosophiques de connaissance. Comme le dit Kholberg $^{13}$ :

To study cognition, one must have some sort of concept of knowledge in terms of which childrens' development is observed... The fact that the cognitive categories of the philosopher are central for understanding the behavior development of the child is so ap-

8. Philosophical Investigations.

9. Concept of Mind.

10. Dreaming.

11. Ibid.

12. FODOR, op. cit.

13. "From Is to Ought ", Cognitive Development and Epistemology (Rédaction, T. Mi). 
parent, once pointed out, that it is only the peculiar epistemology of the positivistic behaviourist which could have obscured it.

Pour le behavioriste, lorsque la " catégorie " clef est l'apprentissage, on considère pour l'apprentissage des opérations logiques ou des "vérités " les mêmes principes que ceux sous-tendant l'apprentissage d'un type de labyrinthe. Mais les erreurs dans un labyrinthe n'impliquent pas de connaissance, elles sont (comme les erreurs dans les psychoses) fausses. Par contre, les vérités logiques sont apprises partiellement en raison de leur vérité. Le fait qu'elles soient apprises dépend du besoin ressenti par l'individu de leur nécessité pour résoudre d'autres dilemmes.

\section{II}

De la même façon que Chomsky nous a démontré que le langage est autre chose qu'un simple comportement verbal, les actes moraux sont autre chose que des particules de comportements observables. Chomsky a souligné l'importance de la compétence dans le langage, c'est-à-dire l'habileté de prononcer et de comprendre un nombre illimité de phrases. Pareillement, nous perdons la richesse d'un comportement moral enfantin en voulant le limiter à une réponse spécifique ou à un nombre déterminé de réponses. $\mathrm{Ce}$ qu'il ne faut jamais ignorer, c'est la situation comme elle lui apparaît à lui-même.

$\mathrm{Si}$ on adhère au mouvement behavioriste, ce qu'on perd surtout c'est la signification de l'acte pour l'acteur lui-même. L'argument apporté par Kohlberg ${ }^{14}$ était que les tests comportementaux utilisés avec les jeunes enfants (tricherie, résistance à la tentation, etc) étaient souvent perçus comme sans importance, et cela justement par les enfants les plus matures. Les tests typiques donnaient aux enfants la pleine opportunité de falsifier leurs résultats, ce que firent $80 \%$ d'entre eux. Toutes ces études semblaient appuyer les deux hypothèses de Hartshorne et de May d'il y a trente ans : a) on ne peut diviser le monde en personnes honnêtes ou malhonnêtes, b) il y a vraiment peu de corrélation entre les résultats obtenus aux tests de situation de tricherie. Dans une étude non typique, citée par Kohlberg, on a démontré qu'après avoir incité les enfants à tricher, même plus que dans les autres tests, seulement

14. Ibid. 
15\% d'entre eux se laissèrent tenter. Kohlberg interpréta ces derniers résultats en considérant la façon qu'avaient les enfants de percevoir leur tâche. En effet, l'instrumentation utilisée comportait une foule de gadgets devant amener d'une façon plus réaliste les enfants à tricher; mais ces derniers crurent qu'elle connaissait leurs véritables résultats et n'osèrent pas les falsifier. On ne peut donc pas conclure sur leur moralité, parce que leur perception de la situation influence leur comportement.

Plusieurs études confirment les vues de Kohlberg concernant les tendances corrélatives entre les résultats aux raisonnements moraux et les indices de tricherie. On a trouvé que parmi trenteneuf étudiants non diplômés, seulement un sur neuf des sujets ayant des "principes" tricha et que parmi ceux qui avaient une moralité " conventionnelle ", la moitié le firent. Une autre de ces études, cette fois avec des enfants de six ans, a démontré que $20 \%$ des enfants du stade supérieur trichèrent, tandis que $67 \%$ de ceux du stade inférieur le firent.

Ce qui nous impressionne le plus, ce sont les comptes rendus des relations entre les raisonnements moraux et le fait de refuser une autorité ordonnant de donner des secousses électriques à une autre personne. Cette étude a été faite non seulement avec des étudiants non gradués de niveau universitaire, mais aussi avec des enfants plus jeunes. Avec des garçons de 11 à 15 ans, on a démontré que plus ils sont confrontés à des raisonnements moraux, plus ils hésitent à punir d'autres personnes. Nous effectuons présentement une étude similaire avec des enfants de 4 à 11 ans, afin de recueillir d'autres données concernant la relation entre le raisonnement moral et le comportement.

De même que les recherches sur les raisonnements moraux, celles concernant les jugements de causalité des enfants doivent tenir compte de l'entière structure de la pensée enfantine. Il est toujours important de sonder (probe). Par exemple, considérons l'épreuve comportementale suivante que nous utilisons présentement dans nos études sur la causalité ; nous nous servons de deux lampes de poche et d'un miroir pour faire apparaître des cercles de lumière sur un mur dans des conditions variables. Comme épreuve comportementale, il s'agit pour l'enfant d'indiquer laquelle des lampes de poche est responsable du cercle de lumière et nous devons enregistrer les résultats en termes de vrai ou faux. Mais on 
ne peut négliger les justifications verbales apportées par l'enfant, car, sans elles, on ne saurait conclure sur la théorie qui sous-tend leurs jugements de causalité. Dans notre expérience, il y a trois situations : la première où les deux lampes de poches sont allumées et dirigées vers le mur ; une deuxième où un miroir empêche le rayon d'une lampe d'atteindre le mur tout en laissant passer celui de la deuxième ; une troisième où la proximité entre en jeu, c'està-dire que la lampe la plus proche du mur n'est pas celle qui est responsable du cercle de lumière. Lorsque l'enfant répond à l'une ou l'autre de ces situations en disant que " la verte " est responsable parce qu'elle est dirigée vers le mur, il faut l'interroger (probe) afin de savoir pourquoi il est important que la lampe soit dirigée vers le mur. Sans ce genre d'interrogation, on ne peut jamais conclure, à savoir si l'enfant a réellement une théorie de la causalité basée sur la transmission d'énergie ou s'il n'utilise que le concept de contiguité spatiale.

Tandis que les tests comportementaux concernant la moralité et la causalité nous apportent des intuitions importantes, ils ne sont pas suffisants pour déterminer le niveau de développement chez l'enfant. Pour la psychologie développementale, l'être humain a des capacités propres à son espèce d'attribuer à lui-même ou aux autres la responsabilité dans des jugements moraux ou causaux. Ces capacités outrepassent la conception behavioriste. Pour le behaviorisme, l'essence de l'acte moral réside dans la " resistence to extinction " et il est vu dans le paradigme d'un chien ou d'un chat qui résiste à la tentation. On utilise d'ailleurs ce dernier par analogie pour les expériences avec les êtres humains. Les formes modernes du behaviorisme (techniques d'entraînement), comme celles de leurs prédécesseurs Watson et Skinner, s'intéressent plus à la prédiction et au contrôle qu'à l'explication. L'approche développementale, par contre, est plus préoccupée par l'explication, et doute que le contrôle, si cher à Skinner, puisse être atteint. On justifie l'emploi de stades du développement par notre incapacité de forcer un enfant à acquérir un nouvel ensemble de concepts en utilisant uniquement les méthodes d'entraînement. L'enfant pourra développer l'habileté de mimer un comportement correspondant à la réponse attendue, mais sera incapable de fournir des raisons et des justifications adéquates pour montrer qu'il a réellement intériorisé le concept en question.

Un certain nombre d'expériences ont démontré que les 
enfants modifiaient leur orientation morale après une période d'entraînement relativement courte. Plusieurs behavioristes prétendent que ces expériences mettent en question les stades du développement ${ }^{15}$. Parmi les plus connues, citons celle de Bandura et de McDonald ${ }^{16}$. Ils ont soumis leurs sujets (garçons entre 5 et 11 ans) à une interaction sociale confrontant leur jugement moral concernant la sévérité de la désobéissance à celui d'un modèle adulte donnant verbalement les raisons sous-tendant ses verdicts toujours contraires. Ils ont montré que, peu importe si les jugements de l'enfant étaient originalement orientés vers les intentions ou les conséquences, il adoptait l'orientation de l'adulte. Bandura soutient que la théorie développementale n'est donc plus nécessaire pour expliquer le niveau de moralité de l'enfant, puisqu'il est adéquatement déterminé en terme de discriminations apprises par l'enfant.

Les piagétiens ont critiqué Bandura pour des raisons méthodologiques, c'est-à-dire qu'il a administré le posttest tout de suite après le traitement expérimental et qu'il a, pour ce faire, utilisé un matériel identique. Il faut admettre cependant que d'autres études plus récentes, dont la nôtre ${ }^{17}$, ont très bien démontré que l'effet de l'entraînement persiste pour quelques semaines et qu'il peut être généralisé pour d'autres situations. Il est important de souligner que la qualité des réponses pendant le posttest pouvait être prédite à l'aide de la théorie développementale.

Il appert qu'un entraînement fonctionnel auprès des enfants de niveau préscolaire réussit à augmenter significativement le niveau d'intentionnalité, mais jamais dans le cas des enfants d'un âge beaucoup plus avancé. Les données nous montrent une hausse d'intentionnalité, mais aussi, une plus grande ambivalence dans leurs jugements et occasionnellement une certaine confusion. Ces conclusions ne nous surprennent nullement, étant donné que nous avions trouvé dans une étude précédente ${ }^{18}$ qu'au cours du développement normal, l'enfant traverse, suite à la pure obéissance à l'autorité, une étape intermédiaire d'ambivalence. On a obtenu des résultats semblables avec des enfants âgés de six (6) ans.

\footnotetext{
15. BANDIIRA, voir no 7 ci-haut.

16. "Influence of social reinforcement and the behaviour of models in shaping children's moral judgments ", Journal of Abnormal and Social Psycbology, 1963, 67, pp. 274-281.

17. "Effects of Training on the Moral Judgment of Young Children ", Journal Personality and Social Psycbology, 1973, vol. 28, no 1, 62-68.

18. Moral Judgments of Cbildren, 1971, thèse doctorale non publiée, Université McGill.
} 
Ces études suggèrent que l'entraînement ne peut jamais reproduire exactement le processus normal de développement. Il y aura toujours des enfants ne pouvant être entraînés, parce qu'ils n'auront pas développé l'équipement conceptuel nécessaire. Cela est confirmé par le fait que dans toutes les études, on retrouve des enfants dont les réponses ne peuvent être améliorées par l'entraînement. En pratique, beaucoup de parents sont notoirement inconsistants en tant que modèles. Leurs enfants sont donc susceptibles d'être assaillis par des moralités de tous les niveaux. Leur capacité d'intérioriser une moralité intentionnelle dépendra en partie de leur statut développemental.

Département des Sciences de l'Éducation, Université du Québec à Montréal. 